\title{
SOME REMARKS ON THE VOLTERRA OPERATOR
}

\author{
CIPRIAN FOIAS AND J. P. WILLIAMS ${ }^{1}$
}

\begin{abstract}
By considering various functions of the Volterra operator $V$, examples are obtained to show that (i) similar contractions need not have the same defect numbers, (ii) unicellular operators may have large spectra, and (iii) the disk $|z| \leqq\|T\|$ need not be a minimal spectral set for an operator $T$ of the form $1+$ compact. A complete description is also given of those disks that are a spectral set for some conjugate of $V$.
\end{abstract}

1. The purpose of this note is to exploit a few simple computations and facts concerning the Volterra operator $V$ defined on $L^{2}(0,1)$ by $V f(x)=$ $\int_{0}^{x} f(t) d t$. These yield a description of those disks that are spectral sets for some conjugate $X^{-1} V X$ of $V$. This in turn is used to obtain some information about the numerical range of $X^{-1} V X$.

By considering various functions of $V$ we also obtain examples of (i) an operator $T$ of the form $1+$ compact for which the disk $|z| \leqq\|T\|$ is not a minimal spectral set, (ii) two similar contractions that have different defect (and hence, an affinity intertwining two contractions that cannot be lifted to an affinity intertwining their unitary dilations), and (iii) a unicellular operator with a large spectrum.

In the statement of some of the results we shall make use of another realization of $V$, namely that in which the Cayley transform $(1-V) \times$ $(1+V)^{-1}$ is unitarily equivalent to the compression operator $S(m)=$ $P S \mid \mathcal{H}(m)$, where $S$ denotes the classical unilateral shift: $S u(\lambda)=\lambda u(\lambda)$ on $H^{2}$, and $P$ is the orthogonal projection of $H^{2}$ onto $\mathscr{H}(m)=$ $H^{2} \ominus m H^{2}$, and $m(\lambda)=\exp (\lambda+1)(\lambda-1)^{-1}$. (See [3] and [10]; see also [14, Chapter IX].)

2. We begin with a simple proof of a result of Kalisch [8]:

Proposition 1. $V$ and $\lambda V$ are similar only if $\lambda=1$.

Received by the editors September 1, 1970.

AMS 1970 subject classifications. Primary 47A25, 47A10; Secondary 47A15, 47A20.

Key words and phrases. Similarity, numerical range, spectral sets, unicellular operators, theory of contractions.

${ }^{1}$ The second author gratefully acknowledges the support of the National Science Foundation. 
Proof. Suppose that $X^{-1} V X=\lambda V$ where $X$ is an affinity. Then (see [8])

$$
\left(\left\|X^{-1}\right\|\|X\|\right)^{-1} \leqq\left\|X^{-1} V^{n} X\right\| /\left\|V^{n}\right\|=|\lambda|^{n} \leqq\|X\|\left\|X^{-1}\right\|
$$

for $n=1,2,3, \cdots$, so that $|\lambda|=1$. Now $(1-\lambda z V)^{-1}=X^{-1}(1-z V)^{-1} X$ for any complex number $z$. Also, for $f \in L^{2}(0,1)$ :

$$
(1-\lambda z V)^{-1} f(x)=f(x)+\lambda z \int_{0}^{x} \exp (\lambda z(x-t)) \cdot f(t) d t .
$$

Hence $\exp (\lambda z x)=X^{-1}(1-z V)^{-1} X(1)$, so that

$$
\|\exp (-\lambda n x)\| \leqq\left\|X^{-1}\right\|\|X\|\left\|(1+n V)^{-1}\right\| \leqq\left\|X^{-1}\right\|\|X\|
$$

for $n \geqq 0$ because $\operatorname{Re} V \geqq 0$. This implies that $\operatorname{Re} \lambda \geqq 0$. Since $\lambda^{k} V=$ $X^{-k} V X^{k}$ for $k \geqq 1$, the same argument shows that $\operatorname{Re} \lambda^{k} \geqq 0$ for $k=$ $1,2,3, \cdots$. This implies $\lambda \geqq 0$. Hence $\lambda=|\lambda|=1$.

3. A closed subset $\sigma$ of the plane is called a spectral set of an operator $T$ on a complex Hilbert space if $\sigma$ contains the spectrum $\sigma(T)$ of $T$ and if $\|u(T)\| \leqq \sup \{|u(z)|: z \in \sigma\}$ for each rational function $u$ with no poles in $\sigma$. J. von Neumann [9] showed that for any complex number $\lambda$ the disk $|z-\lambda| \leqq\|T-\lambda\|$ is a spectral set of $T$. Also, the closed right half-plane $H^{+}$is a spectral set of $T$ if and only if $\operatorname{Re} T \geqq 0$.

PROPOSITION 2. The disk $D_{\lambda}=\{z:|z-\lambda| \leqq|\lambda|\}$ is a spectral set of some conjugate of $V$ if and only if $\lambda>0$.

Proof. Equation (1) implies that if $\alpha$ is a complex number then $(1-\alpha V)^{-1}=1+\alpha M_{\alpha} V M_{\alpha}^{-1}$ where $M_{\alpha}$ denotes the operator of multiplication by $\exp (\alpha x)$ on $L^{2}(0,1)$. Thus $V$ is similar to $M_{\alpha} V M_{\alpha}^{-1}=$ $V(1-\alpha V)^{-1}=V_{\alpha}$ for any complex $\alpha$. Let $\lambda>0$ and let $\alpha=-1 / 2 \lambda$. Then $\left\|V_{\alpha}-\lambda\right\|=\lambda\left\|(1-\alpha V)^{-1}(1+\alpha V)\right\|=\lambda$ because $V$ has positive real part and $0 \in \sigma\left(V_{\alpha}\right)$. The disk $D_{\lambda}$ is therefore a spectral set of $V_{\alpha}$.

Conversely, suppose that $D_{\lambda}$ is a spectral set of some conjugate of $V$. Then there is a positive constant $c$ such that

$$
\left\|\left(1+\frac{1}{\alpha \lambda} V\right)^{-1}\right\| \leqq c \sup \left\{\left.|1+z| \alpha \lambda\right|^{-1}: z \in D_{\lambda}\right\}=\frac{c|\alpha|}{|1+\alpha|-1}
$$

for any complex number $\alpha$ satisfying $|1+\alpha|>1$. The left member of this inequality is at least $\|f\|$ where $f(x)=(1+(1 / \alpha \lambda) V)^{-1} 1=$ $\exp (-x / \alpha \lambda)$. From this it follows easily that $\lambda$ is real (take $\alpha=i t, t$ real) and that $\lambda>0$ (take $\alpha>0)$.

REMARK. In [15] it was shown that any convex neighborhood of the spectrum of an operator $T$ is a spectral set for some conjugate of $T$. This 
fact and Proposition 2 show that if $D$ is a disk, then $D$ is a spectral set for some conjugate of $V$ if and only if either $D$ contains 0 as an interior point, or 0 is a boundary point of $D$ and the center of $D$ lies on the positive real axis.

If $T$ is a nilpotent operator on a finite-dimensional space then $T$ and $\lambda T$ are similar for any complex number $\lambda \neq 0$. In particular, if $S$ is the $n$-dimensional shift with matrix

$$
S=\left(\begin{array}{llll}
0 & & & \\
1 . & . & \\
& \ddots & & \\
& & 1 & 0
\end{array}\right)
$$

then $S$ and $T=\frac{1}{2} S$ are similar. However, rank $\left(1-S^{*} S\right)=1$, rank $\left(1-T^{*} T\right)=n$. Thus the defect number of a contraction is not a similarity invariant. (This remark is due to $R$. G. Douglas.) The next result gives an infinite-dimensional example in which the contractions $S, T$ are irreducible, unicellular, and of norm 1 .

Proposition 3. There exists a contraction $T$ similar to $S(m)$ (see §1) and such that $1-T^{*} T$ and $1-T T^{*}$ are not of finite rank. ${ }^{2}$

Proof. Put $W=V(1+2 V)^{-1}$ and $T=(1-W)(1+W)^{-1}$. Then since $W$ and $V$ are similar, so are $S(m)$ and $T$. Moreover, $T$ is a contraction and

$$
\begin{aligned}
& \operatorname{rank}\left(1-T^{*} T\right) \\
& \operatorname{rank}\left(1-T T^{*}\right)
\end{aligned}=\operatorname{rank} \operatorname{Re} W=\operatorname{rank}\left(V+V^{*}+4 V V^{*}\right)=\infty .
$$

Corollary 1. There exist two similar contractions of class $C_{0}$ (see [14, Chapter III]) that are not unitarily equivalent.

Indeed, the operators $T$ and $S(m)$ above verify these conditions (since $\left.\operatorname{rank}(1-S(m) * S(m))=\operatorname{rank}\left(1-S(m) S(m)^{*}\right)=1\right)$.

COROLlARY 2. There exist two similar completely nonunitary contractions with nonsimilar (minimal, strong) unitary dilations (see [14, Chapter I]).

Proof. The minimal strong unitary dilations of the operators $T$ and $S(m)$ above are the bilateral shifts of multiplicity $\aleph_{0}$, resp. one which are obviously not similar.

REMARK. Recall that if $T_{1}$ and $T_{2}$ are contractions on $\mathcal{H}_{1}$ resp. $\mathcal{H}_{2}$, and if $X$ is an intertwining operator (i.e., $T_{1} X=X T_{2}$ ), and if $U_{1}$ and $U_{2}$ are the minimal strong unitary dilations of $T_{1}$ and $T_{2}$, then there exists an

\footnotetext{
${ }^{2}$ Proposition 3 answers a question raised by Henry Helson in a conversation with the second author.
} 
operator $Y$ such that $U_{1} Y=Y U_{2}$ and $X=P_{1} Y \mid \mathscr{H}_{2}$ where $P_{1}$ denotes the orthogonal projection of the space where $U_{1}$ operates onto $\mathcal{H}_{1}$. Moreover, $Y$ can even be chosen such that $\|Y\|=\|X\|$. (See [14, Chapter II].) A natural question that arises in this context is whether the dilation $Y$ can be chosen to be invertible (i.e., with an everywhere defined bounded inverse) when $X$ is invertible. The negative answer to this question is given by Corollary 2. In fact, if $T_{1}$ and $T_{2}$ are chosen as in Corollary 2, then $T_{1}=X T_{2} X^{-1}$ with an invertible $X$. However $X$ does not have an invertible "dilation" $Y$ since $U_{1}$ and $U_{2}$ are not similar.

4. It is easy to see that a convex spectral set of an operator $T$ must contain the numerical range $W(T)$ of $T$. Therefore Proposition 2 implies that for any positive number $\lambda$ there is an invertible operator $X$ such that $W\left(X^{-1} V X\right) \subset D_{\lambda}$. On the other hand, if $\delta_{\alpha}$ denotes the sector $|\arg z| \leqq$ $\pi / 2 \alpha$, then there does not exist an invertible operator $X$ such that $W\left(X^{-1} V X\right) \subset \mathcal{S}_{\alpha}$, for any $\alpha>1$. (See [12, Theorem 7].) The next result gives some additional negative information about numerical ranges that is useful in the study of problems related to $V$. (In particular, it gives some restrictions on the numerical range of a conjugate of $V$.)

Proposition 4. Let $T$ be a bounded linear operator on a Hilbert space, and suppose that $W(T) \subset H^{+}$. There exists an invertible operator $X$ such that $W\left(X^{-1} T X\right) \subset H^{-}=\left\{z:-z \in H^{+}\right\}$if and only if iT is similar to a selfadjoint operator.

Proof. Let $\psi(z)=(1-z)(1+z)^{-1}$. Then since $H^{+}$is a spectral set for $T$, the unit disk $D=\psi\left(H^{+}\right)$is a spectral set for $\psi(T)$. If $H^{-}$is a spectral set for $X^{-1} T X$, then the exterior of $D\left(=\psi\left(H^{-}\right)\right)$is a spectral set for $\psi\left(X^{-1} T X\right)=X^{-1} \psi(T) X$. These facts imply that $\left\{\left\|\psi(T)^{ \pm n}\right\|: n=1,2\right.$, $3, \cdots\}$ is bounded. Hence, by a well-known theorem of B. Sz.-Nagy [13], $\psi(T)$ is similar to a unitary operator. Hence $i T=i \psi^{-1}(\psi(T))$ is similar to a selfadjoint operator.

COROLlaRY 1. $\mathrm{H}^{-}$cannot contain the numerical range of any conjugate of $V$.

Proposition 4 permits us to show that the similarity of $\alpha V$ and $\alpha V(1-\alpha V)^{-1}$ (see $\left.\S 3\right)$ is not a property of quasi-nilpotent operators as the finite-dimensional case suggests. (It is easy to see that any nilpotent $Q$ is similar to $Q(1-Q)^{-1}$.) Indeed, we have the following:

COROLlaRy 2. There exists a quasi-nilpotent operator $Q$ in the commutant of $V$ such that $Q$ and $Q(1-Q)^{-1}$ are not similar.

To prove this we use the following lemma (pointed out to us by J. G. Stampfli): 
LEMMA. If $Q$ is a nonzero quasi-nilpotent operator such that $\left\|(1+Q)^{-1}\right\| \leqq 1,\left\|(1-Q)^{-1}\right\| \leqq 1$, then $Q$ is not similar to both $Q(1-Q)^{-1}$ and $Q(1+Q)^{-1}$.

Proof. The condition $\left\|(1+Q)^{-1}\right\| \leqq 1$ implies that the numerical range of $Q(1+Q)^{-1}=1-(1+Q)^{-1}$ lies in $H^{+}$. Similarly, $\left\|(1-Q)^{-1}\right\|$ $\leqq 1$ implies that $W\left(Q(1-Q)^{-1}\right) \subset H^{-}$. Thus by Proposition 4, if $Q$ is similar to both $Q(1+Q)^{-1}$ and $Q(1-Q)^{-1}$, then $i Q$ is similar to a selfadjoint operator. Thus $i Q=0, Q=0 .^{3}$

To complete the proof of Corollary 2 it suffices to exhibit an operator $Q$ satisfying the hypothesis of the Lemma. For this purpose let $u$ be a conformal map of the open unit disk onto the region $\Omega \subset H^{+}$bounded by the positive imaginary axis and the circles $|z|=1$ and $\left|z-\frac{1}{3}\right|=\frac{2}{3}$. The map $u$ extends to a homeomorphism (still denoted by $u$ ) from the closed unit disk $D$ onto the closure $\Omega^{-}$of $\Omega$. Obviously we can suppose that $u(1)=1$. From the spectral mapping theorem [2, Chapter I] for von Neumann's functional calculus [9], we deduce $\sigma(u(S(m)))=$ $u(\sigma(S(m)))=u(\{1\})=\{1\}$ so that

$$
Q=2(1-u(S(m)))(1+u(S(m)))^{-1}
$$

is quasi-nilpotent. Moreover, the functions

$$
\left[1 \pm 2 \frac{1-u(\lambda)}{1+u(\lambda)}\right]^{-1}
$$

are in modulus $\leqq 1$ on $D$ which by von Neumann's theorem is a spectral set for $S(m)$. Hence the operators $(1 \pm Q)^{-1}$ are contractions. Finally, $Q \neq 0$. Indeed, if $Q=0$, then $u(S(m))=1$ and this gives (by the composition rule $u(v(T))=(u \circ v)(T)$ for von Neumann's functional calculus [9]) $S(m)=u^{-1} \circ u(S(m))=u^{-1}(1)=1$ which is absurd.

5. In [16] is was shown that if $T$ is a completely nonnormal operator (i.e., without any nontrivial reducing subspace $\mathcal{L}$ such that $T \mid \mathcal{L}$ is normal), and if there is a number $|\alpha|<\|T\|$ such that $T-\alpha$ is compact, then the disk $|z| \leqq\|T\|$ is a minimal spectral set of $T$. We now show that this condition on $\alpha$ is best possible:

Proposition 5. There exists a completely nonnormal operator $A$ of norm 1 such that $A-1$ is compact and such that the unit disk is not a minimal spectral set of $A$.

\footnotetext{
${ }^{3}$ Stampfli's proof of the lemma actually shows that $Q$ is not similar to either of the operators $Q(1 \pm Q)^{-1}$.
} 
Proof. The operator $A=(1+2 V)^{-1}$ is clearly completely nonnormal and $A-1$ is compact. Hence $\|A\| \geqq 1$. Since the right half-plane $H^{+}$is a spectral set of $V$ it follows that $\left\{(1+2 \lambda)^{-1}: \lambda \in H^{+}\right\}=D_{1 / 2}$ is a spectral set of $A$.

6. Another question that can be answered by studying the commutant of $V$ is that of the existence of unicellular operators whose spectra contain more than one point. Indeed, we have the following theorem (whose origin lies in [4, Proposition 2] and in a subsequent suggestion of R. G. Douglas):

Proposition 6. There exists a unicellular operator $W$ whose spectrum contains more than one point.

Proof. Let $G$ be a bounded, simply-connected planar region that is its own Carathéodory hull (see [11]) such that some prime end $E$ of $G$ does not contain an accessible boundary point (see [5, p. 40]). For example, ${ }^{4}$ one can choose for $G$ the region obtained from the unit square $0 \leqq x \leqq 1,0 \leqq y \leqq 1$ by deleting the rectangles

$$
\begin{array}{lll}
1 /(4 n+1) \leqq x \leqq 1 / 4 n, & 1 / n \leqq y \leqq 1 & (n=2,3,4, \cdots) \\
1 /(4 n+3) \leqq x \leqq 1 /(4 n+2), & 0 \leqq y \leqq 1-1 / n & (n=2,3,4, \cdots) .
\end{array}
$$

Let $w$ be a conformal map of the open unit disk onto $G$, and let $W=$ $w(S(m)){ }^{5}$

Now $w$ fails to have a radial limit at the point $\lambda_{0}$ of the unit circle corresponding to $E$, and replacing $w$ by the function $w\left(\lambda_{0} \lambda\right)$ if necessary, we may suppose that $\lambda_{0}=1$. In this case the spectrum of the operator $W$ contains more than one point [4].

On the other hand, a theorem of Farrell [1] implies that the identity function is a weak-star limit of a sequence of polynomials in $w$, i.e., (see [11, Lemma 1]) there exists a sequence $\left\{p_{n}\right\}$ of polynomials such that for $|\lambda|<1$ the sequence $p_{n}(w(\lambda))$ is uniformly bounded and converges to $\lambda$. But then, by the functional calculus for contractions (see [14, Chapter III]), the sequence $p_{n}(W)$ converges in the weak operator topology to $S(m)$. Therefore any invariant subspace for $W$ is also invariant for $S(m)$. Since $S(m)$ is unicellular, so is $W$.

REMARKS. (1) The argument shows that $W$ and $S(m)$ have the same (closed) invariant subspaces. Note also that $W$ is unitarily equivalent to an operator in the commutant $\{V\}^{\prime}$ of $V$.

\footnotetext{
${ }^{4}$ This example was shown to us by G. Schober.

${ }^{5}$ For the functional calculus with $H^{\infty}$ functions see [14, Chapter III].
} 
(2) Proposition 6 yields a negative answer to a conjecture of $P$. Rosenthal (see [6]).

7. We conclude by mentioning two open problems:

(i) It can be shown that either each closed linear invariant subspace of $V$ is hyperinvariant for any $T \in\{V\}^{\prime}$ without eigenvalues, or there exists an operator $T_{0} \in\{V\}^{\prime}$ without nontrivial hyperinvariant subspaces. Which of these possibilities actually holds?

(ii) Must the closure of the numerical range $W(T)^{-}$of a bounded operator $T$ be a spectral set of some conjugate of $T$ ? The answer is affirmative if $W(T)^{-}$is a disk (see [14, Chapter II]) or if $T$ is the Volterra operator (Proposition 2). The answer is also affirmative if the spectrum of $T$ is contained in the interior of the numerical range of $T$ [15], or if the underlying Hilbert space is finite-dimensional (because the eigenvalues in the boundary of $W(T)$ correspond to reducing eigenspaces of $T$ [7] so that the result in [15] applies).

Acknowledgements. The authors thank R. G. Douglas, G. Schober, and J. G. Stampfli for useful discussions and suggestions.

\section{REFERENCES}

1. O. J. Farrell, On approximation to an analytic function by polynomials, Bull. Amer. Math. Soc. 41 (1935), 707-711.

2. C. Foias, Some applications of spectrai sets. 1: Harmonic-spectral measure, Acad. R. P. Romîne. Stud. Cerc. Mat. 10 (1959), 365-401; English transl., Amer. Math. Soc. Transl. (2) 61 (1967), 25-62, MR 22 \#8340.

3. — Modèles fonctionnels, liaison entre les théories de la prédiction, de la fonction caractéristique et de la dilatation unitaire, Deuxième Colloq. l'Anal. Fonct., Centre Belge Recherches Math., Librairie Universitaire, Louvain, 1964, pp. 63-76, MR 32 \#6222.

4. - The class $C_{0}$ in the theory of decomposable operators, Rev. Roumaine Math. Pures Appl. 14 (1969), 1433-1440.

5. G. M. Goluzin, Geometric theory of functions of a complex variable, GITTL, Moscow, 1952; English transl., Transl. Math. Monographs, vol. 26, Amer. Math. Soc., Providence, R.I., 1969. MR 15, 112.

6. P. R. Halmos, Quasitriangular operators, Acta Sci. Math. (Szeged) 29 (1968), 283-293. MR 38 \#2627.

7. S. Hildebrandt, Über den numerischer Wertebereich eines Operators, Math. Ann. 163 (1966), 230-247.

8. G. K. Kalisch, On similarity, reducing manifolds, and unitary equivalence of certain Volterra operators, Ann. of Math. (2) 66 (1957), 481-494. MR 19, 970.

9. J. von Neumann, Eine Spektraltheorie für allgemeine Operatoren eines unitären Raumes, Math. Nachr. 4 (1951), 258-281. MR 13, 254.

10. D. Sarason, A remark on the Volterra operator, J. Math. Anal. Appl. 12 (1965), 244-246. MR 33 \#580.

11. - Weak-star generators of $H^{\infty}$, Pacific J. Math. 17 (1966), 519-528. MR $35 \# 2151$. 
12. J. G. Stampfli and J. P. Williams, Growth conditions and the numerical range in a Banach algebra, Tôhoku Math. J. (2) 20 (1968), 417-424. MR 39 \#4674.

13. B. Sz.-Nagy, On uniformly bounded linear transformations, Acta Univ. Szeged. Sect. Sci. Math. 11 (1947), 152-157. MR 9, 191.

14. B. Sz.-Nagy and C. Foias, Harmonic analysis of operators on Hilbert space, North-Holland, Amsterdam, 1970.

15. J. P. Williams, Similarity and the numerical range, J. Math. Anal. Appl. 26 (1969), 307-314. MR 39 \#2010.

16. - Minimal spectral sets of compact operators, Acta. Sci. Math. (Szeged) 28 (1967), 93-106. MR 36 \#725.

Institute of Mathematics, Academy of R. S. Rumania, Bucharest 12, Rumania

Department of Mathematics, Indiana University, Bloomington, Indiana 47401 